\title{
Combined use of diffusion tensor tractography and multifused contrast-enhanced FIESTA for predicting facial and cochlear nerve positions in relation to vestibular schwannoma
}

\author{
Masanori Yoshino, MD, PhD, ${ }^{1}$ Taichi Kin, MD, PhD, ${ }^{1}$ Akihiro Ito, MD, PhD, ${ }^{1}$ Toki Saito, PhD, ${ }^{2}$ \\ Daichi Nakagawa, MD, ${ }^{1}$ Kenji Ino, PhD, ${ }^{3}$ Kyousuke Kamada, MD, PhD, ${ }^{4}$ Harushi Mori, MD, PhD, ${ }^{3}$ \\ Akira Kunimatsu, MD, PhD, ${ }^{3}$ Hirofumi Nakatomi, MD, PhD, ${ }^{1}$ Hiroshi Oyama, MD, $\mathrm{PhD},{ }^{2}$ and \\ Nobuhito Saito, MD, PhD'
}

Departments of ${ }^{1}$ Neurosurgery, ${ }^{2}$ Clinical Information Engineering, and ${ }^{3}$ Radiology, Graduate School of Medicine, The University of Tokyo, Tokyo, Japan; and ${ }^{4}$ Department of Neurosurgery, Asahikawa Medical University, Asahikawa, Japan

\begin{abstract}
OBJECT The authors assessed whether the combined use of diffusion tensor tractography (DTT) and contrastenhanced (CE) fast imaging employing steady-state acquisition (FIESTA) could improve the accuracy of predicting the courses of the facial and cochlear nerves before surgery.

METHODS The population was composed of 22 patients with vestibular schwannoma in whom both the facial and cochlear nerves could be identified during surgery. According to DTT, depicted fibers running from the internal auditory canal to the brainstem were judged to represent the facial or vestibulocochlear nerve. With regard to imaging, the authors investigated multifused CE-FIESTA scans, in which all 3D vessel models were shown simultaneously, from various angles. The low-intensity areas running along the tumor from brainstem to the internal auditory canal were judged to represent the facial or vestibulocochlear nerve.
\end{abstract}

RESULTS For all 22 patients, the rate of fibers depicted by DTT coinciding with the facial nerve was $13.6 \%$ (3/22), and that of fibers depicted by DTT coinciding with the cochlear nerve was $63.6 \%$ (14/22). The rate of candidates for nerves predicted by multifused CE-FIESTA coinciding with the facial nerve was $59.1 \%(13 / 22)$, and that of candidates for nerves predicted by multifused CE-FIESTA coinciding with the cochlear nerve was $4.5 \%$ (1/22). The rate of candidates for nerves predicted by combined DTT and multifused CE-FIESTA coinciding with the facial nerve was $63.6 \%$ (14/22), and that of candidates for nerves predicted by combined DTT and multifused CE-FIESTA coinciding with the cochlear nerve was $63.6 \%(14 / 22)$. The rate of candidates predicted by DTT coinciding with both facial and cochlear nerves was $0.0 \%$ (0/22), that of candidates predicted by multifused CE-FIESTA coinciding with both facial and cochlear nerves was $4.5 \%$ (1/22), and that of candidates predicted by combined DTT and multifused CE-FIESTA coinciding with both the facial and cochlear nerves was $45.5 \%$ (10/22).

CONCLUSIONS By using a combination of DTT and multifused CE-FIESTA, the authors were able to increase the number of vestibular schwannoma patients for whom predicted results corresponded with the courses of both the facial and cochlear nerves, a result that has been considered difficult to achieve by use of a single modality only. Although the 3D image including these prediction results helped with comprehension of the 3D operative anatomy, the reliability of prediction remains to be established.

http://thejns.org/doi/abs/10.3171/2014.11.JNS14988

KEY WORDS vestibular schwannoma; diffusion tensor tractography; CE-FIESTA; facial nerve; cochlear nerve; diagnostic and operative techniques

\footnotetext{
ABBREVIATIONS 3DRA = 3D rotational angiography; $C E=$ contrast-enhanced; $D T T=$ diffusion tensor tractography; ESE-DNAP = electric stimuli-elicited dorsal cochlear nucleus action potential; FIESTA = fast imaging employing steady-state acquisition; MRA = MR angiography; TOF = time of flight; TRICKS = time-resolved imaging of contrast kinetics; VS = vestibular schwannoma.

SUBMITTED May 2, 2014. ACCEPTED November 26, 2014.

INCLUDE WHEN CITING Published online May 22, 2015; DOI: 10.3171/2014.11.JNS14988.

DISCLOSURE This work was supported in part by Grant-in-Aid for Challenging Exploratory Research (25670618). The authors report no conflict of interest concerning the materials or methods used in this study or the findings specified in this paper.
} 
I $\mathrm{N}$ recent years, prediction of the courses of the facial and vestibulocochlear nerves before surgery for vestibular schwannoma (VS) has been attempted by use of MRI. ${ }^{13,14,18}$ The current gold standard for predicting the locations of the facial and vestibulocochlear nerves in relation to VS is the balanced steady-state free precession method, which includes fast imaging employing steadystate acquisition (FIESTA). ${ }^{12,21}$ However, the number of patients for whom we can predict these locations by using FIESTA decreases with increasing tumor size. ${ }^{15}$ To address this problem, Taoka et al..$^{22}$ used diffusion tensor tractography (DTT) to predict the location of the facial nerve in relation to VS. As this technology has advanced, reports of successful prediction of the location of the facial nerve in relation to large VSs by use of DTT have increased..$^{3,5,17}$ However, various problems have been encountered. First, investigations related to DTT conditions for VS have been insufficient, and the reliability of DTT cannot be considered adequate for everyday clinical application. Second, although some reports have indicated that DTT was able to predict facial nerve location in relation to VS, descriptions of DTT predicting cochlear nerve location in relation to VS have been lacking. With the aim of addressing these issues, we investigated DTT conditions for VS. As a result, we proposed a more reliable method than previously reported $^{24}$ and were able to depict fibers corresponding with the course of the cochlear nerve ${ }^{23}$; however, the rate of depiction of fibers corresponding to the course of the facial nerve was lower than previously reported. In addition, predicting before surgery whether the depicted fibers corresponded with the facial, cochlear, or vestibular nerve or simply represented noise was difficult.

With the study reported here, we assessed whether the combined use of DTT and contrast-enhanced (CE)FIESTA could improve the accuracy of preoperative prediction of the courses of the facial and cochlear nerves. We used multifused CE-FIESTA, with which arteries and veins were shown simultaneously, to completely eliminate surrounding vessels from the tumor. Furthermore, we applied the obtained prediction results to preoperative planning.

\section{Methods \\ Patient Population}

From August 2010 through November 2012, a total of 33 patients underwent surgery for VS in the Department of Neurosurgery at The University of Tokyo Hospital. Of these, 11 patients were excluded from this study because neither the facial nor the cochlear nerve could be identified during surgery. The remaining 22 patients (10 male, 12 female) were included in the study. Mean patient age was 44 years (range 18-64 years). Three of the patients had recurrent VS, and 1 of those patients had neurofibromatosis Type 2. For all patients, preoperative facial nerve function was House-Brackmann Grade I. Preoperative hearing function was Gardner-Robertson ${ }^{4}$ Class 1 for 7 patients, Class 2 for 3 patients, Class 3 for 4 patients, Class 4 for 1 patient, and Class 5 for 7 patients. Tumor size was measured in axial cross-section as the largest diameter parallel to the posterior surface of the petrous bone. Mean tumor size was $29.6 \mathrm{~mm}$ (range $8.6-51.8 \mathrm{~mm}$ ). During surgery, the origin of the tumor was revealed to be the superior vestibular nerve in 8 patients $(36.3 \%)$ and the inferior vestibular nerve in 14 patients $(63.6 \%)$ (Table 1$)$.

The internal review board of The University of Tokyo Hospital approved the study protocol, and written informed consent was obtained from all patients before participation.

\section{Image Acquisition}

MRI was performed by using a Signa 3.0-T system (GE Healthcare) equipped with an 8-channel phased-array head coil. Diffusion tensor images were obtained with a single-shot spin-echo echo-planar sequence by using the following protocol: TR 17,000 msec, TE $65.6 \mathrm{msec}$, slice thickness $2.5 \mathrm{~mm}$ with no gap, FOV $25.6 \mathrm{~cm}$, number of excitations 1 , matrix size $128 \times 128$, and reconstructed images zero-fill interpolated to $256 \times 256$. Diffusion tensor imaging data were acquired along 30 noncollinear gradient directions with a b-value of $1000 \mathrm{sec} / \mathrm{mm}^{2}$ and an additional zero b-image. Realignment of these images and compensation for eddy-current morphing were performed on the basis of the zero b-image on a workstation equipped with the MRI unit. We also acquired anatomical MR images by using FIESTA, CE-FIESTA, time-of-flight (TOF) MR angiography (MRA), CE TOF-MRA, and time-resolved imaging of contrast kinetics (TRICKS). The main imaging parameters for FIESTA and CE-FIESTA were as follows: TR $4.2 \mathrm{msec}$, TE $1.6 \mathrm{msec}$, slice thickness $0.8 \mathrm{~mm}$, FOV $20 \mathrm{~cm}$, matrix size $512 \times 512$, and flip angle $45^{\circ}$. The main imaging parameters for MRA and CE-MRA were as follows: TR $26 \mathrm{msec}$, TE $3 \mathrm{msec}$, slice thickness $0.4 \mathrm{~mm}$, FOV $22 \mathrm{~cm}$, matrix size $512 \times 512$, and flip angle $20^{\circ}$. The main imaging parameters for TRICKS were as follows: TR $3.6 \mathrm{msec}$, TE $1.4 \mathrm{msec}$, slice thickness $1 \mathrm{~mm}$, FOV $24 \mathrm{~cm}$, matrix size $512 \times 512$, and flip angle $20^{\circ}$. A clinical C-arm angiography unit (Allura Xper FD20/10; Philips Medical Systems) was used for 3D rotational angiography (3DRA). The $\mathrm{C}$-arm rotated $240^{\circ}$ at $55^{\circ} / \mathrm{sec}$ and obtained 120 images within a 17-inch FOV during contrast injection. The rotation was initially performed without contrast injection to obtain bone images and later performed with contrast injection. Reconstruction of $3 \mathrm{D}$ volume data was performed with a matrix size of $512 \times 512 \times 512$.

All data were provided as image stacks coded in Digital Imaging and Communication in Medicine format and were processed by using Avizo 6.3 software (Visualization Science Group) and dTV-II SR software ${ }^{11}$ on a personal computer (Dell Precision T7500; CPU Intel Xeon $\times 5550,2.66 \mathrm{GHz}, \mathrm{RAM} 8.00 \mathrm{~GB}$, graphics card NVIDIA Quadro FX5800) for prediction and planning.

\section{Preoperative Prediction by Use of DTT}

We first depicted fibers by using the methods described below. We then determined whether the depicted fibers running from the internal auditory canal to the brainstem represented the facial or the vestibulocochlear nerve (Fig. 1). Depicted fibers that did not reach the brainstem were considered to represent noise. Detailed methods for fiber tracking are as follows. Seed regions of interest were 
TABLE 1. Patient demographic and clinical characteristics

\begin{tabular}{|c|c|c|c|c|c|c|c|c|c|}
\hline \multirow{2}{*}{$\begin{array}{l}\text { Case } \\
\text { No. }\end{array}$} & \multirow{2}{*}{$\begin{array}{c}\text { Age (yrs), } \\
\text { Sex }\end{array}$} & \multirow{2}{*}{$\begin{array}{l}\text { Tumor Size } \\
\quad(\mathrm{mm})\end{array}$} & \multirow{2}{*}{$\begin{array}{l}\text { Second } \\
\text { Surgery }\end{array}$} & \multirow[b]{2}{*}{ NF-2 } & \multirow{2}{*}{$\begin{array}{l}\text { Tumor } \\
\text { Origin }\end{array}$} & \multicolumn{2}{|c|}{ Preoperative Function } & \multicolumn{2}{|c|}{ Postoperative Function } \\
\hline & & & & & & Hearing* & Facial Nerve† & Hearing* & Facial Nerve† \\
\hline 1 & $64, \mathrm{~F}$ & 8.6 & $\mathrm{~N}$ & $\mathrm{~N}$ & SVN & 3 & I & 3 & I \\
\hline 2 & $46, \mathrm{~F}$ & 13.6 & $\mathrm{~N}$ & $\mathrm{~N}$ & IVN & 3 & I & 3 & I \\
\hline 3 & $48, M$ & 17.5 & $\mathrm{~N}$ & $\mathrm{~N}$ & IVN & 1 & I & 2 & I \\
\hline 4 & $43, M$ & 24.2 & $\mathrm{~N}$ & $\mathrm{~N}$ & IVN & 4 & I & 5 & I \\
\hline 5 & $34, \mathrm{M}$ & 31.5 & $\mathrm{~N}$ & $\mathrm{~N}$ & IVN & 1 & I & 1 & I \\
\hline 6 & $52, \mathrm{~F}$ & 20.9 & $\mathrm{~N}$ & $\mathrm{~N}$ & IVN & 1 & I & 1 & I \\
\hline 7 & $63, \mathrm{~F}$ & 42.9 & $\mathrm{~N}$ & $\mathrm{~N}$ & SVN & 5 & I & 5 & I \\
\hline 8 & $41, \mathrm{M}$ & 28.8 & $\mathrm{~N}$ & $\mathrm{~N}$ & IVN & 3 & I & 4 & I \\
\hline 9 & $42, \mathrm{~F}$ & 50.4 & $\mathrm{~N}$ & $\mathrm{~N}$ & SVN & 5 & 1 & 5 & II \\
\hline 10 & $50, M$ & 39.6 & $\mathrm{~N}$ & $\mathrm{~N}$ & IVN & 2 & I & 5 & II \\
\hline 11 & $54, \mathrm{~F}$ & 30.0 & $\mathrm{~N}$ & $Y$ & IVN & 5 & 1 & 5 & 1 \\
\hline 12 & $18, F$ & 40.4 & Y & $\mathrm{N}$ & IVN & 1 & 1 & 5 & I \\
\hline 13 & $36, F$ & 32.0 & $\mathrm{~N}$ & $\mathrm{~N}$ & IVN & 5 & 1 & 5 & II \\
\hline 14 & $34, \mathrm{~F}$ & 27.0 & $\mathrm{~N}$ & $\mathrm{~N}$ & SVN & 1 & 1 & 1 & II \\
\hline 15 & $36, F$ & 51.8 & $Y$ & $\mathrm{~N}$ & SVN & 2 & 1 & 5 & III \\
\hline 16 & $30, \mathrm{M}$ & 28.3 & $\mathrm{~N}$ & $\mathrm{~N}$ & IVN & 3 & 1 & 3 & 1 \\
\hline 17 & $58, \mathrm{M}$ & 36.5 & $\mathrm{~N}$ & $\mathrm{~N}$ & SVN & 5 & 1 & 5 & 1 \\
\hline 18 & $38, \mathrm{~F}$ & 22.1 & $\mathrm{~N}$ & $\mathrm{~N}$ & SVN & 1 & 1 & 1 & I \\
\hline 19 & $40, \mathrm{~F}$ & 24.5 & $\mathrm{~N}$ & $\mathrm{~N}$ & IVN & 1 & I & 1 & I \\
\hline 20 & $49, M$ & 20.1 & $\mathrm{~N}$ & $\mathrm{~N}$ & IVN & 5 & 1 & 5 & II \\
\hline 21 & $49, \mathrm{M}$ & 36.4 & $Y$ & $\mathrm{~N}$ & SVN & 5 & I & 5 & I \\
\hline 22 & $43, \mathrm{M}$ & 28.5 & $\mathrm{~N}$ & $\mathrm{~N}$ & IVN & 2 & 1 & 5 & II \\
\hline
\end{tabular}

IVN = inferior vestibular nerve; $\mathrm{N}=$ no; $\mathrm{NF}-2$ = neurofibromatosis Type $2 ; \mathrm{SVN}=$ superior vestibular nerve; $\mathrm{Y}=$ yes.

* Gardner-Robertson Class.

† House-Brackmann Grade.

placed at the porus of the internal auditory canal in a plane perpendicular to the courses of the facial and cochlear nerves to include all nerves coursing inside the internal auditory canal. No target region of interest was used. For each patient, fractional anisotropy thresholds were set at the upper limit, as the value just before the fibers disappeared. As for other parameters, such as step length and turning angle, we used the default values of dTV-II SR (step length 160; turning angle $30^{\circ}$ ).

\section{Preoperative Prediction by Use of Multifused CE-FIESTA}

Vessel imaging data from modalities such as MRA, CE-MRA, TRICKS, and 3DRA were fused automatically with the normalized mutual information method. We then created a 3D vessel model according to a previously reported method. ${ }^{8-10,25}$ Briefly, to allow 3D visualization of tissue, we selected the modality/sequence that offered the highest contrast for the target tissue. The 3D model was constructed by using a surface-rendering method, which used a model with multiple modalities and multiple thresholds for 1 tissue. For example, for 3D modeling of an artery, the 3D reconstruction was performed with TOFMRA for the thick artery model (main trunk and thick cortical arteries) and with original imaging data from CETOF for the thin artery model. Furthermore, arteries that could not be visualized on MR images were constructed in 3D by using original data from 3DRA. After creation of the 3D vessel model, we investigated multifused CEFIESTA, in which all 3D vessel models were shown simultaneously, from various angles. Low-intensity areas running along the tumor from brainstem to the internal auditory canal were then judged to represent the facial or vestibulocochlear nerve (Fig. 2).

\section{Verification of Predicted Results by DTT and Multifused CE-FIESTA}

After nerves identified intraoperatively were defined as "true," we verified the prediction results. The facial and cochlear nerves were also set as the subjects of verification in this study because only the facial and cochlear nerves could be identified by electrophysiological testing during surgery. That is, for candidate nerves predicted by DTT and multifused CE-FIESTA and the facial and cochlear nerves identified intraoperatively, paths in the central portion of the cistern were classified on the basis of the report by Sampath et al. ${ }^{20}$ as coursing superior, anterior, inferior, posterior, or inside the tumor. In addition, anterior and posterior locations were further subdivided into 3 subcategories: superior one-third, middle one-third, and inferior one-third. When agreement was seen between the pathway area of a candidate nerve predicted by DTT and multifused CE-FIESTA and the pathway of a nerve iden- 

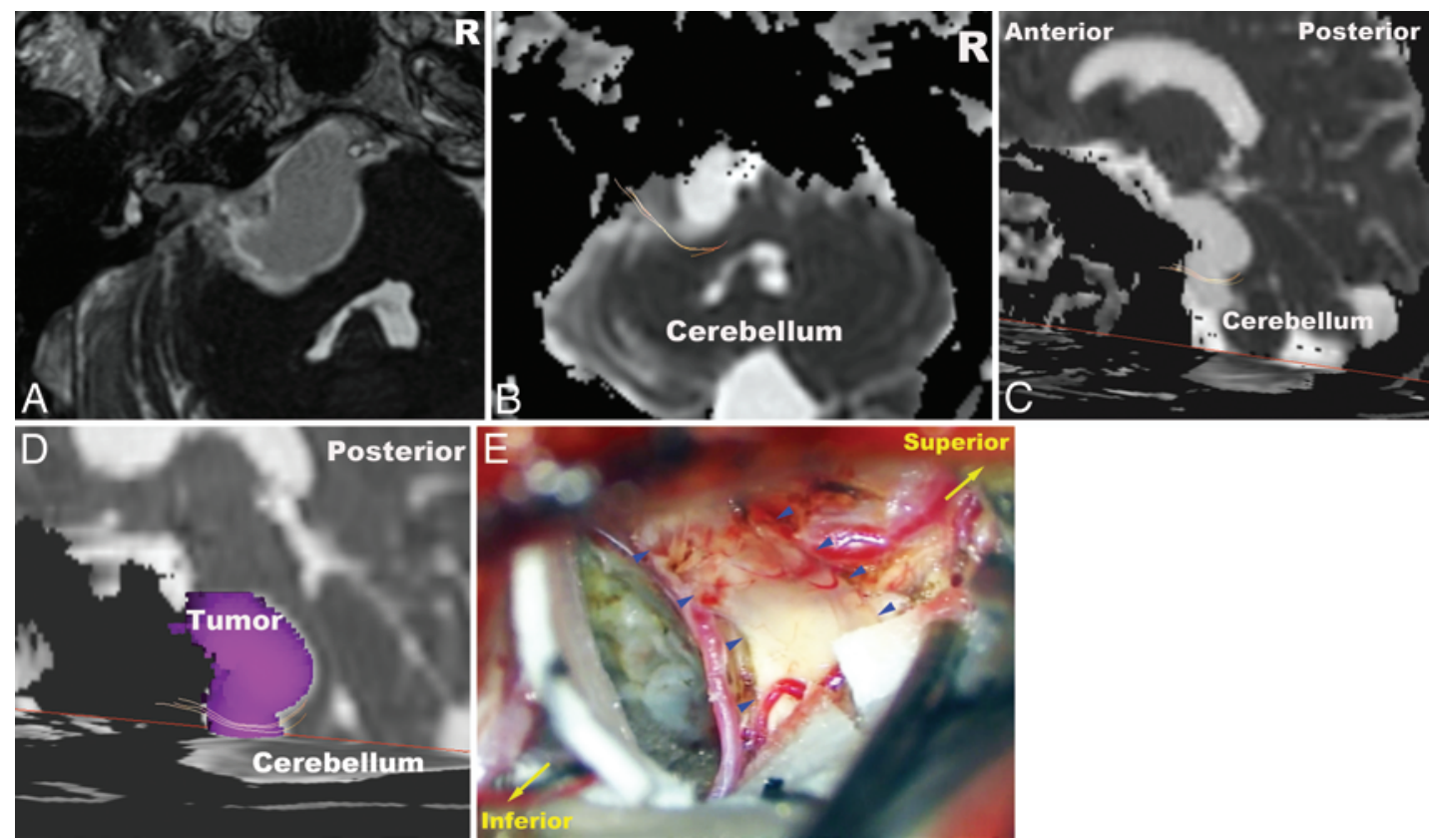

FIG. 1. Case 21. Prediction by DTT. A: CE-FIESTA image revealing a cystic VS at the left cerebellopontine angle. B-D: With the use of DTT (axial $[B]$ and sagittal $[C$ and D] images), fibers are depicted running along the posterior inferior one-third of the tumor surface. E: Electrophysiological diagnosis performed during the surgery confirmed that the cochlear nerve ran along the posterior inferior one-third of the tumor surface (blue arrowheads). $\mathrm{R}=$ right.

tified during surgery, prediction of the nerve by DTT and multifused CE-FIESTA was judged as "correct."

Regarding identification methods for nerves, the facial nerve was identified by the presence of facial muscle action potentials elicited by direct facial nerve fascicle stimulation at $0.1-0.2 \mathrm{~mA}$. With regard to the cochlear nerve, we used our unique invention, electric stimuli elicited dorsal cochlear nucleus action potential (ESE-DNAP) monitor- ing, ${ }^{16}$ to identify the specific direct connection of the specific nerve fascicle with the dorsal cochlear nucleus by intraoperative electrical stimulation at $0.2-0.4 \mathrm{~mA}$ (Video 1 ).

VIDEO 1. Case 10. Intraoperative identification of the facial and cochlear nerves. The facial nerve was identified by facial muscle action potentials elicited by direct facial nerve fascicle stimulation at 0.1-0.2 mA. The cochlear nerve was also identified by the presence of ESE-DNAPs elicited by direct cochlear nerve fascicle
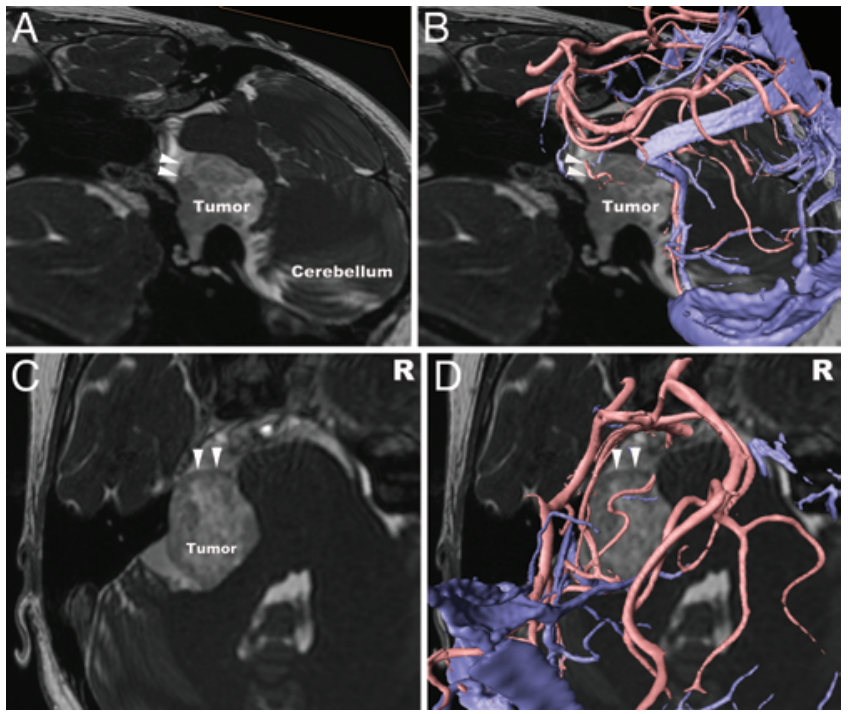

FIG. 2. Case 14. Prediction by multifused CE-FIESTA. A and C: CE-FIESTA images showing several low-intensity areas peripheral to the tumor (arrowheads). A and B: Multifused CE-FIESTA, however, suggests that one low-intensity area is an artery (arrowheads). C and D: In contrast, the images suggest that the other low-intensity area is not a vessel, because no corresponding vessels are evident (arrowheads). The possibility that the latter structure is a nerve is thus considered to be high. 
stimulation at 0.2-0.4 mA. Copyright Masanori Yoshino. Published with permission. Click here to view with Media Player. Click here to view with Quicktime.

In cases in which we could not obtain ESE-DNAPs by intraoperative electrical stimulation, we used macroscopic investigation for identification.

Nerves were intraoperatively identified by the neurosurgeons performing the surgery (N.S., H.N.). Prediction by DTT and multifused CE-FIESTA was performed by author M.Y., and consistency with intraoperative findings was judged by consultation between a neurosurgeon (T.K.) who played no part in the surgery or preoperative DTT visualization and author M.Y.

\section{Preoperative Planning With Predicted Results}

In addition to $3 \mathrm{D}$ vessel models, we also created $3 \mathrm{D}$ brain, nerve, and tumor models from imaging data by using the methods described above. ${ }^{8-10,25}$ The 3D models of the candidates for nerves predicted by DTT and multifused CE-FIESTA were then fused with other 3D brain models, ${ }^{26}$ and all 3D models were visualized on one 3D image. Using this 3D image, we tried to comprehend 3D operative anatomy and investigate how to resect the tumor.

\section{Results}

\section{Outcomes for Facial Nerve and Hearing Functions}

Postoperative facial nerve function was House-Brackmann ${ }^{6}$ Grade I for 15 patients, Grade II for 6 patients, and Grade III for 1 patient. That is, House-Brackmann Grade I-III function was achieved in all 22 patients.

Postoperative hearing function was Gardner-Robertson $^{4}$ Class I for 5 patients, Class II for 1 patient, Class III for 3 patients, Class IV for 1 patient, and Class V for 12 patients. Functional hearing $(<50-\mathrm{dB}$ pure tone average and $>50 \%$ speech discrimination score) was preserved in $9(69.2 \%)$ of 13 patients. For the 8 patients in whom the intraoperative location of the cochlear nerve matched the prediction from combined DTT and multifused CEFIESTA, functional hearing was preserved in 5 patients $(62.5 \%)$.

\section{Intraoperative Locations of the Facial and Cochlear Nerves in Relation to VS}

We located the facial nerve in all patients on the basis of facial muscle action potentials elicited by direct stimulation of facial nerve fascicles. The facial nerve was located on the superior tumor surface in 2 patients, on the anterior superior one-third of the tumor surface in 13 patients, and on the anterior middle one-third of the tumor surface in 7 patients. We also located the cochlear nerve in 14 patients on the basis of ESE-DNAPs elicited by direct stimulation of cochlear nerve fascicles and in 8 patients by macroscopic investigation. The cochlear nerve was located on the anterior middle one-third of the tumor surface in 1 patient, on the anterior inferior one-third of the tumor surface in 15 patients, on the inferior tumor surface in 4 patients, on the posterior inferior one-third of the tumor surface in 1 patient, and on the posterior middle one-third of the tumor surface in 1 patient (Table 2).

\section{Prediction by DTT and Verification of Prediction Results}

Fibers running from the internal auditory canal to the brainstem could be depicted for 18 patients. When we verified the depicted fibers with the true nerves identified intraoperatively, we found agreement between the pathway area of depicted fibers and the pathway area of the facial or cochlear nerves identified during surgery for 17 of these 18 patients. Moreover, at that time, for $3(17.6 \%)$ of the 17 patients for whom fibers could be depicted, the depicted fiber corresponded to the pathway area of the facial nerve, and for $14(82.3 \%)$ of the 17 patients, this corresponded to the pathway area of the cochlear nerve. For all 22 patients, the coincidence rate of fibers depicted by DTT and the facial nerve was $13.6 \%(3 / 22)$, and the that of fibers depicted by DTT and the cochlear nerve was 63.6\% (14/22) (Table 2). According to the above results, the sensitivity of DTT for the facial nerve was $100 \%$, specificity was $20 \%$, positive predictive value was $10 \%$, and negative predictive value was $100 \%$. Positive predictive value indicated those patients for whom depicted fibers agreed with intraoperative findings as a percentage of the total number of patients for whom DTT could predict the nerve. Negative predictive value indicated those patients for whom depicted fibers did not agree with intraoperative findings as a percentage of the total number of patients for whom DTT was able to predict the nerve. In addition, sensitivity of DTT for the cochlear nerve was $100 \%$, specificity was $40 \%$, positive predictive value was $66.7 \%$, and negative predictive value was $100 \%$.

\section{Prediction by Multifused CE-FIESTA and Verification of Predicted Results}

Prediction was possible for 20 patients. When we verified the predicted results with the true nerves identified intraoperatively, we found agreement between the pathway area of a candidate nerve predicted by multifused CE-FIESTA and the pathway area of the facial or cochlear nerves identified during surgery for 13 of 20 patients. Moreover, at that time, in $13(65.0 \%)$ of the 20 patients for whom prediction could be made, the candidate nerve corresponded to the pathway area of the facial nerve, and in $1(5.0 \%)$ patient, the candidate nerve corresponded to the pathway area of the cochlear nerve. As a result, in $1(5.0 \%)$ patient, 2 candidate nerves corresponded to the pathway areas of both the facial and the cochlear nerves, respectively. For all 22 patients, the rate of candidate nerves predicted by multifused CE-FIESTA coinciding with the facial nerve was $59.1 \%$ (13/22), and that of candidate nerves predicted by multifused CE-FIESTA coinciding with the cochlear nerve was $4.5 \%$ (1/22) (Table 2). According to the above results, sensitivity of DTT for the facial nerve was $100 \%$, specificity was $22.2 \%$, positive predictive value was $65 \%$, and negative predictive value was $100 \%$; sensitivity of DTT for the cochlear nerve was $100 \%$, specificity was $1 \%$, positive predictive value was $5 \%$, and negative predictive value was $100 \%$.

\section{Prediction by Combined Use of DTT and Multifused CE-FIESTA and Verification of Predicted Results}

Prediction was possible for 21 patients, and the average 
TABLE 2. Comparison of results predicted by DTT and multifused CE-FIESTA

\begin{tabular}{|c|c|c|c|c|c|c|}
\hline \multirow[b]{2}{*}{ Case No. } & \multicolumn{4}{|c|}{ Intraoperative Findings } & \multicolumn{2}{|c|}{$\begin{array}{c}\text { Prediction Outcomes } \\
\text { (candidate nerve location) }\end{array}$} \\
\hline & $\begin{array}{l}\text { Facial Nerve } \\
\text { Location* }^{*}\end{array}$ & $\begin{array}{l}\text { Presence of } \\
\text { FMAPs }\end{array}$ & $\begin{array}{l}\text { Cochlear Nerve } \\
\text { Location* }\end{array}$ & $\begin{array}{l}\text { Presence of } \\
\text { ESE-DNAPs }\end{array}$ & DTT & CE-FIESTA \\
\hline 1 & AM & Y & $\mathrm{Al}$ & Y & $\mathrm{Al}, \mathrm{I}$ & $\mathrm{AM}, \mathrm{Al}$ \\
\hline 2 & AM & $\mathrm{Y}$ & $\mathrm{Al}$ & $\mathrm{N}$ & - & AM \\
\hline 3 & AS & $\mathrm{Y}$ & AM & Y & AM & AS \\
\hline 4 & AS & Y & $\mathrm{Al}$ & Y & $\mathrm{Al}$ & AS \\
\hline 5 & AS & $\mathrm{Y}$ & $\mathrm{Al}$ & $Y$ & $\mathrm{Al}, \mathrm{PI}$ & AS \\
\hline 6 & $S$ & $\mathrm{Y}$ & PM & Y & PM & AM \\
\hline 7 & AM & $\mathrm{Y}$ & I & $Y$ & I & AM \\
\hline 8 & AS & $Y$ & $\mathrm{Al}$ & $Y$ & $\mathrm{Al}$ & AM, I \\
\hline 9 & AM & Y & $\mathrm{Al}$ & Y & $\mathrm{Al}$ & AM \\
\hline 10 & AM & Y & I & Y & I & AM \\
\hline 11 & AS & Y & $\mathrm{Al}$ & Y & $\mathrm{Al}$ & AS \\
\hline 12 & AS & $Y$ & $\mathrm{Al}$ & $\mathrm{N}$ & AS, I & AS \\
\hline 13 & AS & $Y$ & $\mathrm{Al}$ & $\mathrm{N}$ & - & AM \\
\hline 14 & AM & $Y$ & $\mathrm{Al}$ & $\mathrm{N}$ & 1 & AM \\
\hline 15 & AS & $Y$ & $\mathrm{Al}$ & $Y$ & $\mathrm{Al}$ & AM \\
\hline 16 & AS & $Y$ & $\mathrm{Al}$ & $\mathrm{N}$ & AS & AS \\
\hline 17 & AS & $Y$ & I & $Y$ & 1 & AS \\
\hline 18 & AM & $Y$ & 1 & $Y$ & 1 & - \\
\hline 19 & AS & $Y$ & $\mathrm{Al}$ & $\mathrm{N}$ & - & AM \\
\hline 20 & AS & $Y$ & $\mathrm{Al}$ & $\mathrm{N}$ & AS & AM \\
\hline 21 & $S$ & $Y$ & $\mathrm{PI}$ & $Y$ & $\mathrm{PI}$ & AS \\
\hline 22 & AS & $Y$ & $\mathrm{Al}$ & $\mathrm{N}$ & - & - \\
\hline
\end{tabular}

FMAP = facial muscle action potentials; $-=$ no candidate nerves predicted by DTT or CE-FIESTA.

* Courses of candidate nerves in the central portion of the cistern were classified into the following 9 areas: superior to the tumor (S); anterior superior one-third (AS); anterior middle one-third (AM); anterior inferior one-third (AI); inferior (I); posterior inferior one-third (PI); posterior middle one-third (PM); posterior superior one-third; and inside.

preparation time was 4 hours. When we verified predicted results with the true nerves identified intraoperatively, we found agreement between the pathway area of a candidate nerve predicted by DTT or multifused CE-FIESTA and the pathway area of the facial or cochlear nerves identified during surgery for $18(85.7 \%)$ of 21 patients. Moreover, at that time, in $14(66.7 \%)$ of the 21 patients for whom a prediction could be made, the candidate nerve corresponded to the pathway area of the facial nerve, and in 14 patients (66.7\%), the candidate nerve corresponded to the pathway area of the cochlear nerve. For $10(47.6 \%)$ of these 21 patients, candidate nerves corresponded to the pathway areas of both the facial and the cochlear nerves. For all 22 patients, the rate of candidate nerves predicted by the combination of DTT and multifused CE-FIESTA coinciding with the facial nerve was $63.6 \%$ (14/22), and that of candidate nerves predicted by combining DTT and multifused CE-FIESTA coinciding with the cochlear nerve was $63.6 \%$ (14/22). In addition, the rate of candidates predicted by DTT coinciding with both the facial and cochlear nerves was $0.0 \%(0 / 22)$, that of candidates predicted by multifused CE-FIESTA coinciding with both the facial and cochlear nerves was $4.5 \%$ (1/22), and that of candidates predicted by combined DTT and multifused CE-FIESTA coinciding with both the facial and cochlear nerves was 45.5\% (10/22) (Table 2). According to the above results, sensitivity of DTT for the facial nerve was $100 \%$, specificity was $12.5 \%$, positive predictive value was $66.7 \%$, and negative predictive value was $100 \%$; sensitivity of DTT for the cochlear nerve was $100 \%$, specificity was $12.5 \%$, positive predictive value was $66.7 \%$, and negative predictive value was $100 \%$.

\section{Illustrative Case Case 10}

This patient was a 50-year-old man with hearing loss and a left-sided VS. The maximum diameter of the tumor was $39.6 \mathrm{~mm}$. DTT, applied first for prediction, depicted fibers running inferior to the tumor and extending from the internal auditory canal to the brainstem (Fig. 3A). Next, multifused CE-FIESTA showed a low-intensity area running along the anterior middle one-third of the tumor surface, which was predicted to be the nerve (Fig. 3B). Using 3D imaging including these predicted results, we were able to gain a clear understanding of the 3D operative anatomy (Fig. 3C). 


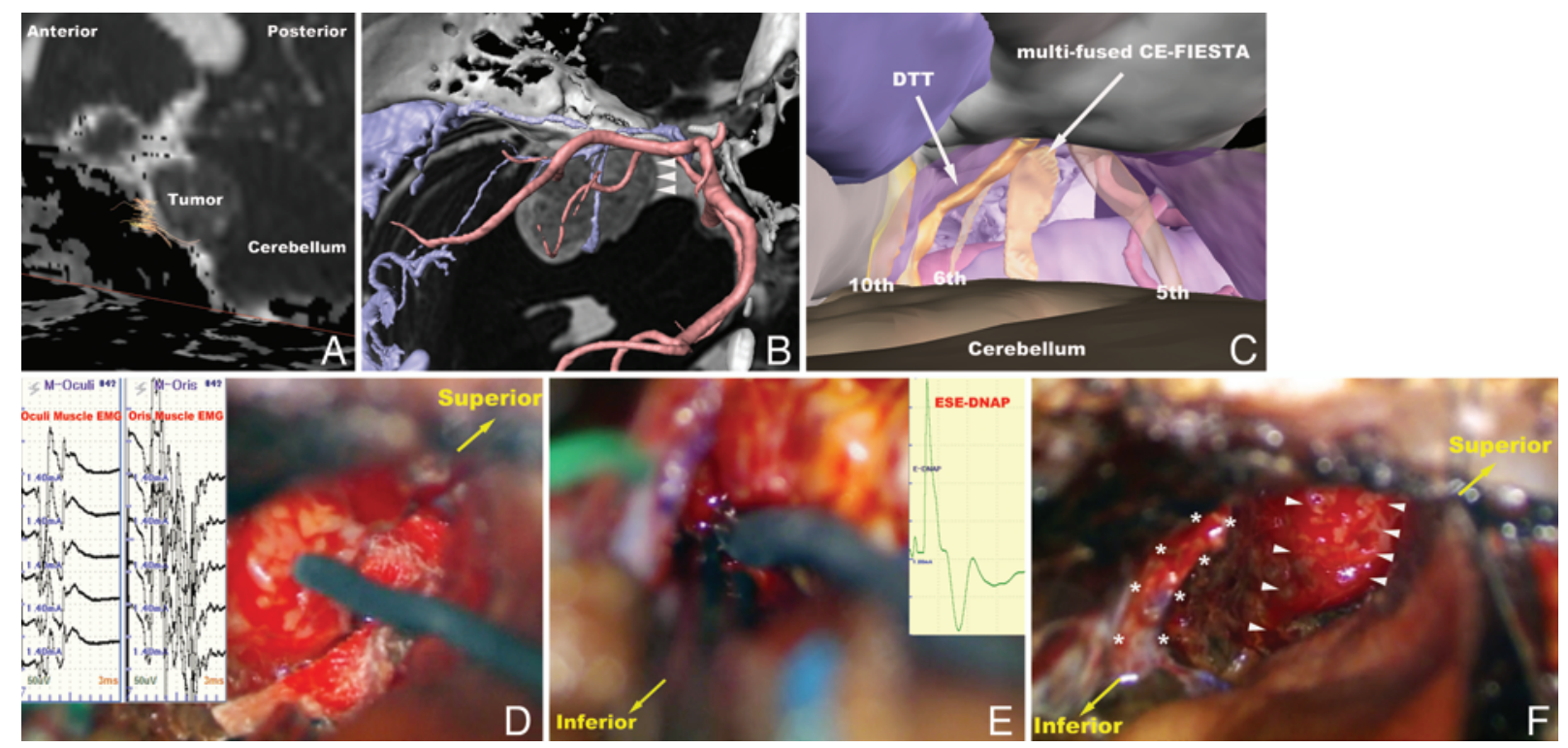

FIG. 3. Case 10. Prediction by combined DTT and multifused CE-FIESTA. A: With the use of DTT, fibers are depicted running inferior to the tumor. B: Multifused CE-FIESTA image revealing a low-intensity area in the anterior middle one-third of the tumor surface (arrowheads). C: 3D imaging including predicted results enables clear comprehension of the 3D operative anatomy. D: Facial muscle action potentials were obtained by monopolar stimulation of the anterior middle one-third of the tumor. E: ESE-DNAPs were obtained by monopolar stimulation inferior to the tumor. F: The pathway area of fibers depicted by DTT corresponds to the pathway area of the cochlear nerve identified intraoperatively (asterisk). The pathway area of the candidate nerve predicted by multifused CE-FIESTA corresponds to the pathway area of the facial nerve identified intraoperatively (arrowheads). EMG = electromyogram.

Surgery was performed using a lateral suboccipital approach. Electrophysiological diagnosis performed during the surgery confirmed that the facial nerve ran along the anterior middle one-third of the tumor surface, and the cochlear nerve passed inferior to the tumor (Fig. 3D-F, Video 1). These results showed that the pathway area of the fibers depicted by DTT corresponded with the actual pathway area of the cochlear nerve and that the pathway area of the candidate nerve predicted by multifused CEFIESTA corresponded with the actual pathway area of the facial nerve.

\section{Discussion}

In this study, the combined use of DTT and multifused CE-FIESTA enabled prediction of both the facial and the cochlear nerve locations in relation to VS in 10 of 22 patients with VS (mean tumor size $29.6 \mathrm{~mm}$ ). In addition, using 3D images based on these results, we were able to gain a clear comprehension of the 3D operative anatomy.

\section{Prediction by Multifused CE-FIESTA}

The resolution of FIESTA images depends on T2 (spinspin relaxation time)/T1 (spin-lattice relaxation time), ${ }^{1}$ and contrast enhancement with media such as gadolinium has a shortening effect on $\mathrm{T} 1$. As a result, contrast between the cranial nerve and the tumor is enhanced. ${ }^{2}$ CE-FIESTA has therefore been used to improve the accuracy of predicting nerve locations in relation to tumor. ${ }^{12}$ However, distinguishing nerves and small vessels on CE-FIESTA was sometimes difficult because small vessels were not always enhanced by contrast medium. This difficulty was thought to decrease prediction accuracy. As a result, to enable the elimination of surrounding small vessels from the tumor and improve prediction accuracy in this study, we fused vessel images by using CE-FIESTA. As a result, candidate nerves predicted by multifused CE-FIESTA corresponded with the facial nerve identified during surgery for 13 (59.1\%) of 22 patients with VS (mean tumor size $29.6 \mathrm{~mm}$ ). This result was superior to previously reported results, ${ }^{15}$ in which the location of the facial nerve in relation to VS was predicted for $38(46.3 \%)$ of 82 patients (mean tumor size $26.4 \mathrm{~mm}$ ). Elimination of surrounding small vessels improved the accuracy of nerve location predictions.

In contrast, we found limitations of this method in terms of the accuracy of the 3D vessel model and the accuracy of registration between the 3D vessel model and CE-FIESTA. With regard to the accuracy of the 3D vessel model, we took care to depict all surrounding vessels 3 -dimensionally in reference to the original images. As for registration accuracy, to decrease image misalignment, at the time of vessel image fusion, we restricted the whole area of the image to the area we wanted to investigate ${ }^{26}$ : the area peripheral to the VS. Because of these efforts, critical errors were not encountered in this study. However, continued investigation of whether this method is valid seems warranted, given that this method was only applied to 22 patients.

\section{Prediction by Combined Use of DTT and Multifused CE-FIESTA}

In this study, when DTT alone was used, the pathway area of depicted fibers corresponded with the pathway area 
of the cochlear nerve for 14 of 22 patients. Conversely, when multifused CE-FIESTA alone was used, the pathway area of the candidate for nerve predicted by multifused CE-FIESTA corresponded with the pathway area of the facial nerve for 13 of 22 patients. As mentioned above, the preoperative prediction of nerve location was limited by the use of only 1 modality. However, these results also show that the fibers depicted by DTT corresponded more frequently with the cochlear nerve than with any other nerves, and the candidate nerve predicted by multifused CE-FIESTA corresponded more frequently with the facial nerve than with any other nerves. In this way, each modality showed a different prediction trend, so prediction of the location of both the facial and cochlear nerves could be achieved for 10 of 22 patients. The location of both the facial and cochlear nerves could not previously be predicted for patients with a VS larger than $15 \mathrm{~mm} .^{12}$ These results might therefore be 1 of the reasons why the rates of functional facial nerve preservation and functional hearing preservation in patients for whom nerve locations could be predicted by combined use of DTT and multifused CEFIESTA (100\% and $62.5 \%$, respectively) was superior to previously reported results (rates of functional facial nerve preservation $81 \%^{19}$ and $96 \%$ and rates of functional hearing preservation $51 \%{ }^{19}$ and $42.5 \%$, respectively ${ }^{7}$ ). Although our findings were superior to previous findings, overall rates of functional facial nerve preservation and functional hearing preservation were $100 \%$ and $69.2 \%$, respectively, so improvements in intraoperative electrophysiological monitoring and operative technique might enable higher rates of functional hearing preservation to be achieved. However, because our study included only 22 patients, continued investigation to clarify the influence of these predictions to clinical outcome seems to be needed.

The reasons for the difference in prediction results between DTT and multifused CE-FIESTA are as follows. First, the cochlear nerve is changed to an extremely thin structure as a result of being compressed by the tumor. In contrast, the facial nerve maintains a bundle structure because it is separate from the origin of the tumor (the vestibular nerve). FIESTA can therefore identify the location of the facial nerve more easily than that of the cochlear nerve. Second, this study set the fractional anisotropy threshold at the upper limit for each patient; thus, nerve fiber tracts with higher fractional anisotropy values can be depicted at a higher rate than those with lower fractional anisotropy values. In addition, when we used DTT for VS patients, the nerve fiber tract depicted by DTT as the cochlear nerve presumably included part of the vestibular nerve because the cochlear nerve cannot be separated from the vestibular nerve at the brainstem and cerebellopontine segment. As a result, the fractional anisotropy values of the nerve fiber tract depicted as the cochlear nerve were higher than those of the facial nerve, leading to a higher rate of cochlear nerve depiction by DTT. Continued investigation to clarify reasons for such discrepancies seems warranted.

\section{Application of 3D Images to Preoperative Planning}

By using the combination of DTT and multifused CEFIESTA, we were able to create detailed 3D images, including exact nerve locations. Our 3D images enabled us to comprehend not only locations of the facial and cochlear nerves but also brain and vessels peripheral to the VS. Exact comprehension of the 3D operative anatomy by use of our 3D images was thought to help reduce the risk for intraoperative complications.

\section{Study Limitations}

In this study, the pathway area of candidate nerves predicted by using the combination of DTT and multifused CE-FIESTA corresponded with the pathway areas for both the facial and cochlear nerves for 10 of 22 patients. However, whether the prediction represented the facial or cochlear or some other nerve was difficult to predict preoperatively. When we could predict 2 candidates, we could estimate which was the facial nerve and which was the cochlear nerve on the basis of the course and positional relationship of nerves discovered by intraoperative investigation. ${ }^{20}$ However, the situation was uncertain, and when we predicted only 1 candidate, we based our estimations on the course and positional relationship. In this study, prediction tendencies for DTT and multifused CE-FIESTA seemed to differ, but whether the tendency was specific to each modality was uncertain. Investigation of how to distinguish prediction results must therefore continue.

\section{Conclusions}

In this research, by using the combination of DTT and multifused CE-FIESTA, we were able to increase the numbers of VS patients for whom predicted results corresponded with the courses of both the facial and cochlear nerves, a result that has been considered difficult when only a single modality is used. Although our 3D images including these prediction results helped us to comprehend the $3 \mathrm{D}$ operative anatomy, the reliability of prediction remains to be established.

\section{Acknowledgment}

We thank Minoru Tanaka for suggestions for the investigation.

\section{References}

1. Amano Y, Takahama K, Nozaki A, Amano M, Kumazaki T: Magnetic resonance portography using contrast-enhanced fat-saturated three-dimensional steady-state free precession imaging. J Magn Reson Imaging 19:238-244, 2004

2. Amemiya S, Aoki S, Ohtomo K: Cranial nerve assessment in cavernous sinus tumors with contrast-enhanced 3D fastimaging employing steady-state acquisition MR imaging. Neuroradiology 51:467-470, 2009

3. Chen DQ, Quan J, Guha A, Tymianski M, Mikulis D, Hodaie M: Three-dimensional in vivo modeling of vestibular schwannomas and surrounding cranial nerves with diffusion imaging tractography. Neurosurgery 68:1077-1083, 2011

4. Gardner G, Robertson JH: Hearing preservation in unilateral acoustic neuroma surgery. Ann Otol Rhinol Laryngol 97:55-66, 1988

5. Gerganov VM, Giordano M, Samii M, Samii A: Diffusion tensor imaging-based fiber tracking for prediction of the position of the facial nerve in relation to large vestibular schwannomas. J Neurosurg 115:1087-1093, 2011

6. House JW, Brackmann DE: Facial nerve grading system. Otolaryngol Head Neck Surg 93:146-147, 1985

7. Jacob A, Robinson LL Jr, Bortman JS, Yu L, Dodson EE, 
Welling DB: Nerve of origin, tumor size, hearing preservation, and facial nerve outcomes in 359 vestibular schwannoma resections at a tertiary care academic center. Laryngoscope 117:2087-2092, 2007

8. Kin T, Nakatomi H, Shojima M, Tanaka M, Ino K, Mori H, et al: A new strategic neurosurgical planning tool for brainstem cavernous malformations using interactive computer graphics with multimodal fusion images. J Neurosurg 117:78-88, 2012

9. Kin T, Oyama H, Kamada K, Aoki S, Ohtomo K, Saito N: Prediction of surgical view of neurovascular decompression using interactive computer graphics. Neurosurgery 65:121129, 2009

10. Kin T, Shin M, Oyama H, Kamada K, Kunimatsu A, Momose T, et al: Impact of multiorgan fusion imaging and interactive 3-dimensional visualization for intraventricular neuroendoscopic surgery. Neurosurgery 69 (1 Suppl Operative):ons40-ons48, 2011

11. Masutani Y, Aoki S, Abe O, Hayashi N, Otomo K: MR diffusion tensor imaging: recent advance and new techniques for diffusion tensor visualization. Eur J Radiol 46:53-66, 2003

12. Mikami T, Minamida Y, Yamaki T, Koyanagi I, Nonaka T, Houkin K: Cranial nerve assessment in posterior fossa tumors with fast imaging employing steady-state acquisition (FIESTA). Neurosurg Rev 28:261-266, 2005

13. Mitsuoka H, Arai H, Tsunoda A, Okuda O, Sato K, Makita $\mathrm{J}$ : Microanatomy of the cerebellopontine angle and internal auditory canal: study with new magnetic resonance imaging technique using three-dimensional fast spin echo. Neurosurgery 44:561-567, 1999

14. Naganawa S, Koshikawa T, Fukatsu H, Ishigaki T, Fukuta T: MR cisternography of the cerebellopontine angle: comparison of three-dimensional fast asymmetrical spin-echo and three-dimensional constructive interference in the steadystate sequences. AJNR Am J Neuroradiol 22:1179-1185, 2001

15. Nakai T, Yamamoto H, Tanaka K, Koyama J, Fujita A, Taniguchi M, et al: Preoperative detection of the facial nerve by high-field magnetic resonance imaging in patients with vestibular schwannoma. Neuroradiology 55:615-620, 2013

16. Nakatomi H, Miyazaki H, Tanaka M, Kin T, Yoshino M, Oyama $\mathrm{H}$, et al: Improving functional preservation in acoustic neuroma surgery. J Neurosurg 122:24-33, 2015

17. Roundy N, Delashaw JB, Cetas JS: Preoperative identification of the facial nerve in patients with large cerebellopontine angle tumors using high-density diffusion tensor imaging. J Neurosurg 116:697-702, 2012

18. Ryu H, Tanaka T, Yamamoto S, Uemura K, Takehara Y, Isoda H: Magnetic resonance cisternography used to determine precise topography of the facial nerve and three components of the eighth cranial nerve in the internal auditory canal and cerebellopontine cistern. J Neurosurg 90:624-634, 1999

19. Samii M, Gerganov V, Samii A: Improved preservation of hearing and facial nerve function in vestibular schwannoma surgery via the retrosigmoid approach in a series of 200 patients. J Neurosurg 105:527-535, 2006

20. Sampath P, Rini D, Long DM: Microanatomical variations in the cerebellopontine angle associated with vestibular schwannomas (acoustic neuromas): a retrospective study of 1006 consecutive cases. J Neurosurg 92:70-78, 2000

21. Shigematsu Y, Korogi Y, Hirai T, Okuda T, Ikushima I, Sugahara T, et al: Contrast-enhanced CISS MRI of vestibular schwannomas: phantom and clinical studies. J Comput Assist Tomogr 23:224-231, 1999

22. Taoka T, Hirabayashi H, Nakagawa H, Sakamoto M, Myochin K, Hirohashi S, et al: Displacement of the facial nerve course by vestibular schwannoma: preoperative visualization using diffusion tensor tractography. J Magn Reson Imaging 24:1005-1010, 2006

23. Yoshino M, Kin T, Ito A, Saito T, Nakagawa D, Ino K, et al: Feasibility of diffusion tensor tractography for preoperative prediction of the location of the facial and vestibulocochlear nerves in relation to vestibular schwannoma. Acta Neurochir (Wien) [epub ahead of print], 2015

24. Yoshino M, Kin T, Ito A, Saito T, Nakagawa D, Kamada $\mathrm{K}$, et al: Diffusion tensor tractography of normal facial and vestibulocochlear nerves. Int J Comput Assist Radiol Surg 10:383-392, 2014

25. Yoshino M, Kin T, Nakatomi H, Oyama H, Saito N: Presurgical planning of feeder resection with realistic three-dimensional virtual operation field in patient with cerebellopontine angle meningioma. Acta Neurochir (Wien) 155:1391-1399, 2013

26. Yoshino M, Kin T, Saito T, Nakagawa D, Nakatomi H, Kunimatsu A, et al: Optimal setting of image bounding box can improve registration accuracy of diffusion tensor tractography. Int J Comput Assist Radiol Surg 9:333-339, 2014

\section{Author Contributions}

Conception and design: Yoshino. Acquisition of data: Yoshino, Ino, Nakatomi, Saito. Analysis and interpretation of data: Yoshino, Ito, Saito, Mori, Kunimatsu. Drafting the article: Yoshino. Critically revising the article: Yoshino, Ito, Saito, Mori, Oyama, Saito. Reviewed submitted version of manuscript: Yoshino, Kin, Ito, Saito, Nakagawa, Kamada, Mori, Kunimatsu, Nakatomi, Oyama, Saito. Administrative/technical/material support: Kin, Nakatomi. Study supervision: Saito.

\section{Supplemental Information \\ Videos}

Video 1, Media Player. http://mfile.akamai.com/21490/wmv/ digitalwbc.download.akamai.com/21492/wm.digitalsource-naregional/jns14-988_video_1.asx.

Video 1, Quicktime. http://mfile.akamai.com/21488/mov/ digitalwbc.download.akamai.com/21492/qt.digitalsource-global/ jns14-988_video_1.mov.

\section{Correspondence}

Masanori Yoshino, Department of Neurosurgery, Graduate School of Medicine, The University of Tokyo, 7-3-1 Hongo, Bunkyo-ku, Tokyo 113-8655, Japan. email: ymasa-tky@umin. ac.jp. 